\title{
Electronic Structure of Silicon-Based Nanostructures
}

\author{
G. G. Guzmán-Verri ${ }^{1,2}$ and L. C. Lew Yan $\operatorname{Voon}^{1}$ \\ ${ }^{1}$ Department of Physics, Wright State University, \\ 3640 Colonel Glenn Highway, Dayton, Ohio 45435, USA \\ ${ }^{2}$ Centro de Investigación en Ciencia e Ingeniería de Materiales, \\ Universidad de Costa Rica, 2060 San José, Costa Rica
}

(Dated: July 4, 2011)

\begin{abstract}
We have developed an unifying tight-binding Hamiltonian that can account for the electronic properties of recently proposed Si-based nanostructures, namely, Si graphene-like sheets and Si nanotubes. We considered the $s p^{3} s^{*}$ and $s p^{3}$ models up to first- and second-nearest neighbors, respectively. Our results show that the Si graphene-like sheets considered here are metals or zerogap semiconductors, and that the corresponding Si nanotubes follow the so-called Hamada's rule [Phys. Rev. Lett. 68, 1579 1992]. Comparison to a recent ab initio calculation is made.

PACS numbers: 73.21.La, 02.60.Cb
\end{abstract}

\section{INTRODUCTION}

After the first synthesis of carbon nanotubes (CNT's) by Iijima more than a decade ago, ${ }^{1}$ other types of nanotubes have been predicted and experimentally observed such as GaN, BN, and AlN among others..$^{2-5}$ However, it was not until fairly recently that the most obvious alternative candidate for creating graphene-like sheets and nanotubes was proposed: $\mathrm{Si}^{6}{ }^{6}$ In addition, different structures have been proposed, each one with different hybridizations: $s p^{2}, s p^{2}-s p^{3}$ and $s p^{3}$. So far, most studies agree that the $s p^{2}$ configuration is the least favorable one and, in contrast, the $s p^{3}$ configuration is one of the most favorable ones because of stability reasons. ${ }^{6-9}$

On the experimental side, there are now at least six independent reports of the fabrication of silicon nanotubes in the laboratory. ${ }^{10-15}$ On the theoretical side, only a handful of papers have explored the electronic properties of these nanomaterials; moreover, practically all of them correspond to $a b$ initio calculations. ${ }^{6-9,16}$ The above early work was reviewed by Perepichka and Rosei. ${ }^{17}$

In the present work, we apply tight-binding (TB) models, so successfully used to study the electronic properties of graphene and CNT's, ${ }^{18}$ to the Si nanostructures that have $s p^{2}$ and $s p^{3}$ hybridization. We will refer to these structures as silicene, Si (111), Si hexagonal nanotubes (Si h-NT's), and Si gear-like nanotubes (Si g-NT's). Silicene is a two dimensional sheet with a honeycomb lattice of lattice constant $a$ made out of Si atoms which have $s p^{2}$ hybridization. Thus, silicene has the same structure as a graphene sheet but it is composed of $\mathrm{Si}$ atoms instead of $\mathrm{C}$ atoms. A Si (111) layer has a lattice structure which is the same as the honeycomb lattice for silicene, except that one set of atoms (e.g., B) is vertically displaced (e.g., down) from the A-plane due to the $s p^{3}$ bonding (see Fig. 1). Single-walled Si h-NT's and Si g-NT's are formed by rolling up, respectively, a silicene and a $\mathrm{Si}$ (111) sheet.

Our goal is two-fold. First, we would like to compare the electronic properties of silicene and $\mathrm{Si}$ (111) to graphene, and the electronic properties of Si h-NT's and Si g-NT's to CNT's. Second, inconsistencies between an $a b$ initio calculation and a proposed $\pi$-TB model, both by Yang and $\mathrm{Ni}^{7}$ motivated us in developing a coupled $\sigma-\pi$ TB model. Results on the effect of the coupling will be presented.

The present paper is organized as follows: in Section II we describe the TB theory for the Si nanostructures, in Section III we present and discuss our results, and in Section IV we give the conclusions. An appendix has been added for further details about the model in question.

\section{THE TIGHT-BINDING MODEL}

We now present a new theory of the band structure of a single Si sheet. This theory applies to both the flat $\mathrm{Si}$ sheet and the Si (111) layer.

The reason a unifying Hamiltonian is possible is because the lattice structure of a $\mathrm{Si}$ (111) sheet is similar to graphene. Figure 1(a) is a two-dimensional representation of the lattice in question. The $\mathrm{A}$ atoms are in the $x y$-plane and the $\mathrm{B}$ atoms are out of it and located at $z=-a /(2 \sqrt{6})$ (the $z$ axis points towards the reader). Thus, the word sheet in the present work means one atomic plane of $\mathrm{A}$ atoms above one atomic plane of $\mathrm{B}$ atoms, i.e., a sheet is two atomic planes. The basis vectors of the lattice are $\mathbf{a}_{1}=(a / 2)(\sqrt{3},-1)$ and $\mathbf{a}_{2}=(a / 2)(\sqrt{3}, 1)$ with magnitude $a .^{18}$ If we label as $l$ the Si-Si bond distance, then $a=l / \sqrt{2}$. We will see that the choice of this coordinate system facilitates the transition from the $\mathrm{Si}$ (111) sheet to the silicene one. The shaded area corresponds to the two-dimensional unit cell of Si (111). Notice that the basis vectors and the unit cell of $\mathrm{Si}$ (111) are equal to the ones in the honeycomb lattice of graphene. ${ }^{18}$ Due to this similarity with graphene, the Brillouin zone of the $\mathrm{Si}$ (111) lattice is the same as the one of graphene.

In order to compute the band structure of $\mathrm{Si}(111)$, we use a first-nearest neighbor (1NN) $s p^{3} s^{*}$ and a secondnearest neighbor (2NN) $s p^{3}$ orthogonal tight-binding model. For these two models, we derive their respective $10 \times 10$ and $8 \times 8$ Hamiltonians. These choices were dic- 


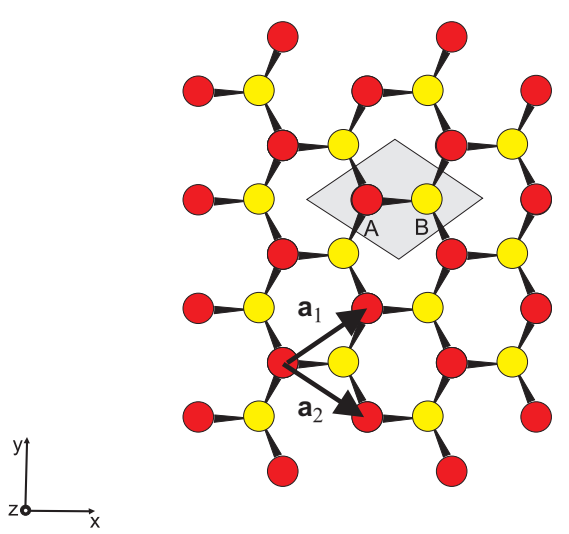

(a)

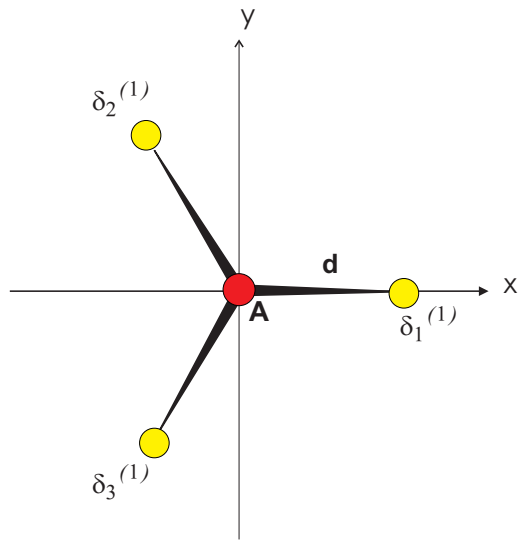

(b)

FIG. 1. (Color online) Lattice of Si (111). (a) Two-dimensional representation of a Si (111) sheet. The atoms labeled as A are all in the $x y$ plane $\left(z_{A}=0\right)$ and all the $\mathrm{B}$ atoms are located below the plane $[z=-a /(2 \sqrt{6})]$. Hence, the sheet is composed by two atomic planes: one of $\mathrm{A}$ atoms and another of $\mathrm{B}$ atoms. The A plane is above the B plane. Notice that the $z$ axis points towards the reader. The vectors $\mathbf{a}_{1}$ and $\mathbf{a}_{2}$ are the two-dimensional basis vectors and the shaded area is the Si (111) unit cell. (b) First-nearest neighbors of Si (111).

TABLE I. Silicon two-center parameters obtained from Vogl et al. ${ }^{19}$ and Grosso and Piermarocchi. ${ }^{20}$ Blank spaces correspond to parameters that do not belong to the model.

\begin{tabular}{lrr}
\hline \hline Parameter & Vogl et al. ${ }^{19}$ & Grosso and Piermarocchi. \\
\hline$E_{s}$ & -4.2000 & -4.0497 \\
$E_{p}$ & 1.7150 & 1.0297 \\
$E_{s^{*}}$ & 6.6850 & \\
$(s s \sigma)_{1}^{A B}$ & -2.0750 & -2.0662 \\
$(s p \sigma)_{1}^{A B}$ & 2.4808 & 2.0850 \\
$(p p \sigma)_{1}^{A B}$ & 2.7163 & 3.1837 \\
$(p p \pi)_{1}^{A B}$ & -0.7150 & -0.9488 \\
$\left(s^{*} p \sigma\right)_{A B}^{1}$ & 2.3274 & \\
$(s s \sigma)_{2}^{A A}$ & & 0.0000 \\
$(s p \sigma)_{2}^{A A}$ & & 0.0000 \\
$(p p \sigma)_{2}^{A A}$ & & 0.8900 \\
$(p p \pi)_{2}^{A A}$ & & -0.3612 \\
\hline \hline
\end{tabular}

tated by the availability of good Si TB parameters which correspond to Vogl et al. ${ }^{19}\left(s p^{3} s^{*}\right)$ and to Grosso and Piermarocchi ${ }^{20}\left(s p^{3}\right)$. The authors are aware of newer TB parametrizations, ${ }^{21,22}$ however, these parametrizations do not reproduce well the Si bulk bands along the $K \Gamma$ direction, which is important for the Si nanostructures.

Like silicene and graphene, the wavevector $\mathbf{k}$ of $\mathrm{Si}$ (111) in the Hamiltonian is two dimensional, however, their dispersion functions $g_{j}(\mathbf{k})$, differ due to the $\sigma-\pi$ coupling (see Appendix A). Furthermore, we use the twocenter approximation (TCA) in order to obtain the angular dependence in the TB parameters. For the sake of completeness, the TCA parameters are reproduced in Table I. We first specify the position vectors of the $1 \mathrm{NN}$ for Si (111):

$$
\begin{aligned}
\boldsymbol{\delta}_{1}^{(1)} & =\left(\frac{a}{\sqrt{3}}, 0,-\frac{a}{2 \sqrt{6}}\right), \\
\boldsymbol{\delta}_{2}^{(1)} & =\left(-\frac{a}{2 \sqrt{3}}, \frac{a}{2},-\frac{a}{2 \sqrt{6}}\right), \\
\boldsymbol{\delta}_{3}^{(1)} & =\left(-\frac{a}{2 \sqrt{3}},-\frac{a}{2},-\frac{a}{2 \sqrt{6}}\right),
\end{aligned}
$$

and for the $2 \mathrm{NN}$ :

$$
\begin{array}{ll}
\boldsymbol{\delta}_{1}^{(2)}=(0, a, 0), & \boldsymbol{\delta}_{2}^{(2)}=(0,-a, 0) \\
\boldsymbol{\delta}_{3}^{(2)}=\left(\frac{a \sqrt{3}}{2},-\frac{a}{2}, 0\right), & \boldsymbol{\delta}_{4}^{(2)}=\left(-\frac{a \sqrt{3}}{2}, \frac{a}{2}, 0\right), \\
\boldsymbol{\delta}_{5}^{(2)}=\left(\frac{a \sqrt{3}}{2}, \frac{a}{2}, 0\right), & \boldsymbol{\delta}_{6}^{(2)}=\left(-\frac{a \sqrt{3}}{2},-\frac{a}{2}, 0\right) .
\end{array}
$$

For simplicity, Fig. 1(b) only shows the position vectors of the $1 \mathrm{NN}$. Notice that the $x$ - and $y$-components of the $1 \mathrm{NN}$ in Si (111) correspond to the ones of graphene. The non-zero $z$-components are the vertical displacements of the $B$ atoms of the Si (111) sheet. In other words, the choice of the coordinate system facilitates the transition from $\mathrm{Si}$ (111) and silicene by making the $z$ component of the position vectors in Eq. (1) equal to zero. The $2 \mathrm{NN}$ coincide for both sheets.

The dispersion $E(\mathbf{k})$ for the $\mathrm{Si}(111)$ sheet is shown in Figs. 2(d) and 2(e). For the $s p^{3}$ model, we can find ana- 
lytic formulae for the dispersion relation at the $\Gamma$ point,

$$
\begin{aligned}
& E_{p+}(\Gamma)=E_{p}+3\left[(p p \sigma)_{2}^{A A}+(p p \pi)_{2}^{A A}\right] \\
&+ \frac{1}{3}\left[4(p p \sigma)_{1}^{A B}+5(p p \pi)_{1}^{A B}\right], \\
& E_{p-}(\Gamma)=E_{p}+3\left[(p p \sigma)_{2}^{A A}+(p p \pi)_{2}^{A A}\right] \\
&-\frac{1}{3}\left[4(p p \sigma)_{1}^{A B}+5(p p \pi)_{1}^{A B}\right],
\end{aligned}
$$

where $E_{p \pm}$ is twofold degenerate.

Next, we explain how this formalism developed for Si (111) can be transfered to silicene.

The $\pi$ and $\sigma$ bands in silicene can be obtained from the previous Hamiltonian by making the $z$-component of the $1 \mathrm{NN}$ equal to zero and substituting the appropriate direct cosines of the $1 \mathrm{NN}$ position vectors. As a result, the $\pi$ and $\sigma$ bands are decoupled, as in graphene. This allow us to consider the bands independently.

For the $\pi$ bands, we recover the well known $2 \times 2$ Hamiltonian but now including $2 \mathrm{NN}$ interactions,

$$
H(\mathbf{k})=\left[\begin{array}{cc}
E_{p}+(p p \pi)_{2}^{A A} g_{25}(\mathbf{k}) & \gamma_{0} g_{12}(\mathbf{k}) \\
\gamma_{0} g_{12}^{*}(\mathbf{k}) & E_{p}+(p p \pi)_{2}^{A A} g_{25}(\mathbf{k})
\end{array}\right]
$$

where $\gamma_{0}\left(=\left|(p p \pi)_{1}^{A B}\right|\right)$ is the transfer integral and the $g_{j}(\mathbf{k})$ functions are given in Eq. (A3). The energy dispersion relation for silicene is found from Eq. (4),

$$
E(\mathbf{k})=E_{p}+(p p \pi)_{2}^{A A} g_{25}(\mathbf{k}) \pm(p p \pi)_{1}^{A B} w(\mathbf{k}),
$$

where the dispersion function $w(\mathbf{k}) \equiv\left|g_{12}(\mathbf{k})\right|=$ $\sqrt{1+4 \cos \frac{\sqrt{3} k_{x} a}{2} \cos \frac{k_{y} a}{2}+4 \cos ^{2} \frac{k_{y} a}{2}}$. Notice that if we make the $2 \mathrm{NN}$ contribution equal to zero, i.e., $(p p \pi)_{2}^{A A}=$ 0 , we recover graphene's energy dispersion in the $1 \mathrm{NN}$ approximation. ${ }^{18}$

For the $\sigma$ bands, the matrix elements of the Hamiltonian correspond to similar ones given in Eq. (A2) for Si (111), but now the $g_{j}(\mathbf{k})$ functions correspond to the ones given in Eq. (A4). These bands are shown in Figs. 2(a) and 2(b).

By evaluating the Hamiltonian at the $\Gamma$ point, we can obtain analytical formulae for the dispersion relations,

$$
\begin{aligned}
E_{p_{z} \pm}(\Gamma)=E_{p} & \pm 3(p p \pi)_{1}^{A B}, \\
E_{s \pm}(\Gamma)=E_{s} & +6(s s \sigma)_{2}^{A A} \pm 3(s s \sigma)_{1}^{A B}, \\
E_{p \pm}(\Gamma)=E_{p} & +3\left[(p p \sigma)_{2}^{A A}+(p p \pi)_{2}^{A A}\right] \\
& \pm \frac{3}{2}\left[(p p \pi)_{1}^{A B}+(p p \sigma)_{1}^{A B}\right],
\end{aligned}
$$

where $E_{p \pm}(\Gamma)$ is twofold degenerate.

Finally, the Si h-NT and Si g-NT band structures are computed from Si (111) and silicene by imposing boundary conditions on $\mathbf{k}$ along the chiral direction. ${ }^{18}$ The results are shown in Fig. 3.

We now proceed to examine our findings obtained from the TB theory.

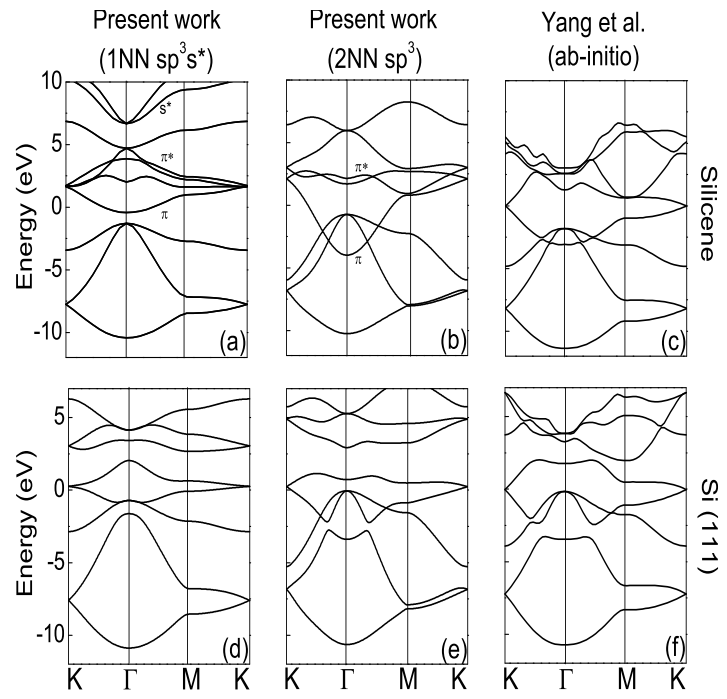

FIG. 2. Band structure of silicene and of Si (111) obtained from our TB models compared to the ab-initio results from Yang and $\mathrm{Ni}^{7}$

\section{RESULTS AND DISCUSSION}

The electronic band structures for the Si sheets and the Si-NT's are shown in Figs. 2 and 3. Here, we discuss them separately.

\section{A. Silicene}

The silicene band structure is shown in Figs. 2(a) and 2(b) according to the $s p^{3} s^{*}$ and $s p^{3}$ models, respectively. Like in graphene, the silicene $\pi$ bands are not coupled to the $\sigma$ bands due to the planar and orbital symmetries. ${ }^{18}$ When we compare the $\pi$ energy bands of silicene from the $s p^{3} s^{*}$ model to the corresponding ones in graphene, we notice that they have a similar form. This occurs because both sheets have the same lattice structure. As far as the $\sigma$ bands are concerned, the valence bands in silicene have been lowered down, therefore, the crossings that occur in graphene between the $\pi$ and $\sigma$ bands do not occur in silicene. Different results are obtained when we perform the same comparison using the $s p^{3}$ model. The $\pi$ band maintains the form as in graphene, nevertheless, the $\pi^{*}$ band changes its curvature as it approaches $\Gamma$ from the $K \Gamma$ and the $M \Gamma$ directions. This change could occur because of the $2 \mathrm{NN}$ interactions or because of the signs of the TB parameters. In order to determine which is the case, one has to perform further calculations on the curvature of the band, which will be presented somewhere else. In contrast to the $s p^{3} s^{*}$ model, the $\pi$ and $\sigma$ valence bands do cross. This shows how sensitive the band structure is to 


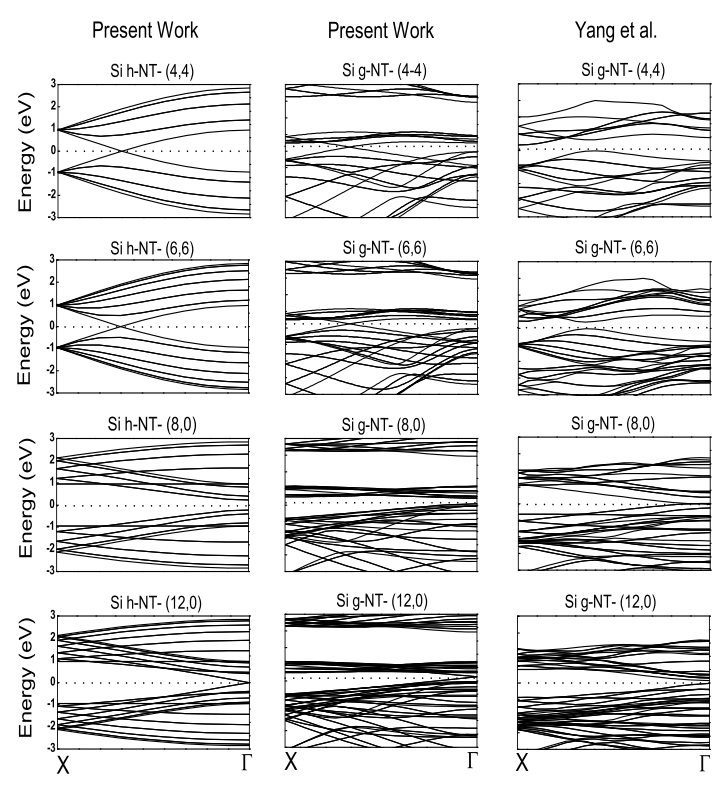

FIG. 3. Band structures of Si h- and Si g-NT's according to our calculations and to Yang and Ni. ${ }^{7}$ The two-center parameters used in our calculations were taken from Grosso and Piermarocchi.$^{20}$

the TB parametrization of the model.

It is known that close to the $K$ point, graphene shows a linear dispersion, ${ }^{23-26}$

$$
E(p)= \pm v_{0}|p|,
$$

where $v_{0}$ and $p(=\hbar k)$ are the Fermi velocity and the carrier momentum, respectively. In graphene, it has been measured that $v_{0} \approx 10^{6} \mathrm{~m} / \mathrm{s}^{23,26}$ The linearity of $E(p)$ is responsible for the electrons to behave as Dirac massless fermions. ${ }^{23,24}$ Figures 2(a) and 2(b) show that silicene has a linear dispersion close to the $K$ point. By performing a linear fitting, we find that the Fermi velocity $v_{0}$ in Eq. (6) is of the order of $10^{5} \mathrm{~m} / \mathrm{s}$ for both models. Compared to graphene, the electrons move slower in silicene. This occurs since the $\pi$ bonds, which are the responsible for conduction in the sheets, are weaker in silicene than in graphene (the atomic distance in silicene is greater than in graphene).

Next, let us now compare our $s p^{3} s^{*}$ results to Yang's $a b$-initio ones. The band structure of silicene obtained by Yang and $\mathrm{Ni}^{7}$ is shown in Fig. 2(c). When compared one notices certain differences. For example, in the neighborhood of the $\Gamma$ point in Fig 2(a), both conduction and valence bands have the opposite curvature with respect to Fig. 2(c). Possibly, this difference can be attributed to the $1 \mathrm{NN}$ approximation in the $s p^{3} s^{*}$ model. There is agreement in the silicene $\pi$ and the lower $\sigma$ bands, however, this is not the case for the upper $\pi^{*}$ band, which has opposite curvature. Moreover, there are no crossings between the $\pi$ and $\sigma$ valence bands. As it was discussed previously, this is due to the TB parameters.

\section{B. Si (111)}

The band structure of $\mathrm{Si}$ (111) is shown in Fig. 2(d) and 2(e) for the $s p^{3} s^{*}$ and the $s p^{3}$ models, respectively. In $\mathrm{Si}$ (111), the $s p^{3}$ hybridization causes a coupling between the $\pi$ and the $\sigma$ states. The effect of it is evident in the anti-crossing of the originally uncoupled bands in graphene. ${ }^{18}$ Note, however, that the $\pi$ band is still doubly degenerate at the $K$ point. In graphene, the degeneracy at this point is required by the hexagonal symmetry. ${ }^{27}$ When we lower the B atoms in silicene to create the Si (111) sheet, as it is shown in Fig. 4, this symmetry is not removed hence, the $\pi$ bands are still degenerate at $K$ in Si (111).

Notice that the $s p^{3}$ and the $s p^{3} s^{*}$ models lead to different bands, particularly, close to the $\Gamma$ point. As the bands approach this point, the curvature of some of them changes. Consider, for instance, the second-lowest valence band in Figs. 2(d) and 2(e). Close to $\Gamma$, this band has negative curvature in the former figure, while it has positive curvature in the latter one. These differences might be due to $2 \mathrm{NN}$ interactions. We computed the band structure of Si (111) using two parameter sets for a $s p^{3}$ TB model by Chadi and Cohen; ${ }^{28}$ one of them for $1 \mathrm{NN}$ only, and the other one for $1 \mathrm{NN}$ plus one $2 \mathrm{NN}$ interaction. These results are not presented here, however, we mention that the band without the $2 \mathrm{NN}$ interaction has negative curvature, like Fig. 2(d), and the band with only one $2 \mathrm{NN}$ interaction has positive curvature, like Fig. 2(e). Another possible reason for the curvature differences is the sign of the TB parameters. Further calculations on the curvature of the bands at the $\Gamma$ point are needed in order to determine if it is due to the $2 \mathrm{NN}$ interactions or the TB parameters. These calculations will be shown somewhere else.

As far as the eigenstates are concerned, they are different in graphene and in Si (111). The $\pi$ bands in both structures are a good example of this. Whereas the eigenstates of the the $\pi$ bands in graphene are $p_{z}$ orbitals, the eigenstates of the $\pi$ bands in $\mathrm{Si}$ (111) correspond to a linear combination of $s, p_{x}, p_{y}$ and $p_{z}$ orbitals. This occurs because in $\mathrm{Si}$ (111), the $\pi$ and $\sigma$ bands are coupled.

Notice in Figs. 2(d) and 2(e) that close to the $K$ point, the dispersion relation is linear, which indicates the presence of Dirac massless fermions. By performing a linear fitting, we find that the Fermi velocity $v_{0}$ is of the order of $10^{4} \mathrm{~m} / \mathrm{s}$. Compared to graphene, the Dirac fermions move slower in the $\mathrm{Si}$ (111) sheet. In order to understand the difference in velocities, let us look at the hybridizations of both sheets, as it is shown in Fig 4 .

On the one hand, each atom shows $s p^{2}$ hybridization in graphene. In this hybridization, each atom has a lobe that is perpendicular to the sheet plane (the sheet corresponds to the $x y$-plane and the lobe is oriented along 


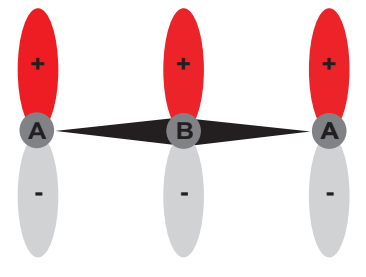

(a)

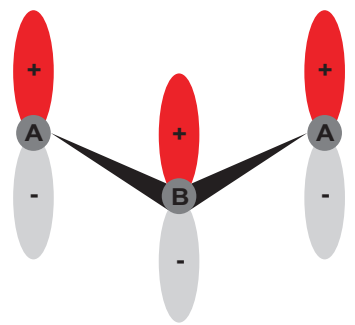

(b)
FIG. 4. (Color online) The $s p^{2}$ and $s p^{3}$ hybridizations in (a) silicene and in (b) Si (111).

the positive $z$-axis as it is shown in Fig. 4(a)). All lobes are oriented along the same direction, i.e., the $z$-axis, and, therefore, they form $\pi$ bonds with their 1NN. The $\pi$ bonds are responsible for the conducting character of the sheet.

On the other hand, Si (111) shows $s p^{3}$ hybridization. Figure 4(b) shows that each atom has a lobe that is perpendicular to the sheet, however, neighboring atoms have their lobes pointing in opposite directions, i.e., the positive and negative $z$-axes. Due to the alternating orientation, a lobe that points along the, say, positive $z$-axis, will not form a $\pi$ bonding with its $1 \mathrm{NN}$, but with its $2 \mathrm{NN}$.

Since the $\pi$ bonds in graphene occur between $1 \mathrm{NN}$, the coupling is stronger than in Si (111), where the bonds occur between 2NN. An electron finds it "easier" to tunnel from one atom to the another one when the coupling is stronger. This explains why electrons in graphene move faster than they do in $\mathrm{Si}$ (111).

The band structure of the Si (111) sheet computed by Yang and $\mathrm{Ni}^{7}$ is shown in Fig. 2(f). When compared to our $s p^{3}$ findings, we find good agreement between them, especially, along the $K \Gamma M$ directions. Close to $\Gamma$, the curvature problem is overcame by including some TCA interactions from $2 \mathrm{NN}$ in the Hamiltonian, namely, $(p p \sigma)_{2}^{A A}$ and $(p p \pi)_{2}^{A A}$. Most of the differences occur along the $M K$ direction, for instance, our calculations show that the second-lowest valence band should have the opposite curvature when it is compared to Yang and $\mathrm{Ni}^{7}$ The positive curvature of this band comes from the strong repulsion induced by the $p$-like band located above it.

\section{Si h-NT}

Nanotubes are fully characterized by their chiral vector $\mathbf{C}_{h}=n \mathbf{a}_{1}+m \mathbf{a}_{2}$, where $n$ and $m$ are integer numbers, and $\mathbf{a}_{1}$ and $\mathbf{a}_{2}$ are given above. ${ }^{18}$ The band structures of Si h-NT's with chiral vectors $(4,4),(6,6),(8,0)$ and $(12,0)$ are shown in the left-most column of Fig. 3 . The dashed line corresponds to the Fermi level. These bands were obtained by substituting the quantized nanotube wave vector $\mathbf{k}$ in Eq. (5). In this figure, the dispersion $g_{25}(\mathbf{k})$, introduced by the $2 \mathrm{NN}$ interactions, its hardly noticeable, therefore, the band structure of Si h-NT's looks very similar to the one of CNT's in the $1 \mathrm{NN}$ approximation. It is due to this similarity, that we neglect the dispersion $g_{25}(\mathbf{k})$ in the band structure of Si h-NT's and discuss it as if it were a $1 \mathrm{NN}$ approximation only. In the $1 \mathrm{NN}$ case, the only difference between the CNT and the $\mathrm{Si}$ h-NT band structures is a scaling factor, which corresponds to the transfer integral $\gamma_{0}\left(\gamma_{0}=-3.033 \mathrm{eV}\right.$ for $\mathrm{C}$ and $\gamma_{0}=-0.949 \mathrm{eV}$ for $\mathrm{Si}$ ). Whether or not a Si h-NT would be conductor or semiconductor, does not depend on $\gamma_{0}$ but on its symmetries, thus, Si h-NT will have similar electronic properties as CNT's, i.e., if $n-m$ is a multiple of 3 , the nanotube is a metal, otherwise, it is a semiconductor. We will refer to this property as Hamada's rule. ${ }^{29}$ From Hamada's rule, we conclude that all armchair Si h-NT's are conductors $(n=m)$ and zig zag tubes are conductors if $n$ is a multiple of $3(m=0)$. The band structures for Si h-NT's shown in Fig. 3 confirm this rule.

We proceed to compare the energy band gaps between CNT's and Si h-NT's. According to Dresselhaus et al. ${ }^{30}$ the band gap is given by

$$
E_{g}=2 \gamma_{0} \operatorname{Min} w\left(k \frac{\mathbf{K}_{2}}{K_{2}}+\frac{1}{3}(N \pm 1) \mathbf{K}_{1}\right)
$$

where $\mathbf{K}_{1}$ and $\mathbf{K}_{2}$ are the reciprocal vectors of graphene and silicene, $\gamma_{0}$ is the transfer integral, $N$ is the number of hexagons in the nanotube unit cell, $k$ is the wave vector, and Min $w$ is the minimum of the graphene and silicene dispersion relation $w\left(=\left|g_{12}(\mathbf{k})\right|\right)$ with respect to $k$. In particular, for zig zag tubes, the minimum occurs at $k=$ $0,{ }^{18}$ according to Eq. (7), this yields to,

$$
E_{g}=2 \gamma_{0} \sqrt{1+4 \cos \frac{\pi q_{0}}{n}+4 \cos ^{2} \frac{\pi q_{0}}{n}},
$$

where $q_{0}=(1 / 3)(N \pm 1)$ and $N=2 n$. In Fig. 5, we show our results for the band gap of both CNT's and h-SiNT's as a function of the diameter. For a given diameter, the band gap $E_{g}$ of Si h-NT's is smaller than the band gap of CNT's. In order to understand this result, we should look to the approximate expression for $E_{g}$ derived by Saito et al. ${ }^{18}$ and Ando, ${ }^{24}$

$$
E_{g} \sim \frac{|\gamma|}{d}
$$

where $\gamma=\sqrt{3} a \gamma_{0} / 2$ and $d=\left|\mathbf{C}_{h}\right| / \pi$ (the nanotube diameter). For a given diameter, $E_{g}$ depends only on the parameter $\gamma$, which depends on the transfer integral (recall that the transfer integral corresponds to the interaction between neighboring $p_{z}$ orbitals). Since this interaction is smaller for Si h-NT's than for CNT's, the band gap is greater for the latter.

Notice that the $1 / d$ dependence in the nanotube band gap is expected. If we let $d \rightarrow \infty$, then $E_{g} \rightarrow 0$. The limit 


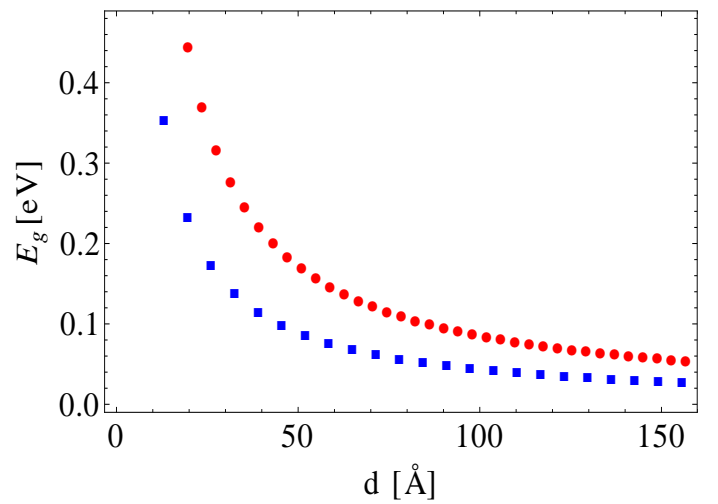

FIG. 5. (Color online) Band gap of CNT's (circles) and Si h-NT's (squares) as a function of their diameters.

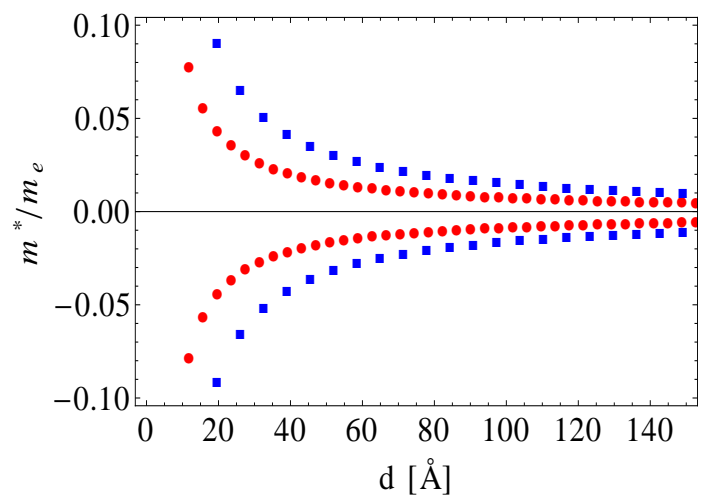

FIG. 6. (Color online) Effective masses of CNT's (circles) and Si h-NT's (squared) as a function of their diameters.

$d \rightarrow \infty$ corresponds to making a nanotube thicker and thicker, and, therefore, more similar to graphene, which has a zero band gap.

We proceed to compute and to compare the effective masses of Si h-NT's and CNT's. By differentiating the band structure of zig zag nanotubes given in Eq. (5), and neglecting the $2 \mathrm{NN}$ interactions, we obtain an analytical formula for their effective masses at the $K$ point,

$$
\begin{aligned}
m_{ \pm}^{*} & =\left.\frac{\hbar^{2}}{d^{2} E / d k^{2}}\right|_{k=0} \\
& = \pm \frac{2 \hbar^{2}}{3 \gamma_{0} a^{2}} \frac{\sqrt{1+4 \cos \frac{\pi q_{0}}{n}+4 \cos ^{2} \frac{\pi q_{0}}{n}}}{\cos \frac{\pi q_{0}}{n}} .
\end{aligned}
$$

Equation (10) is plotted in Fig. 6. First, notice the general trend for Si h-NT's and CNT's that the effective masses decrease as their diameter increases. This occurs because electrons in a $\mathrm{Si}$ h-NT (CNT) with large diameter, behave more similar to electrons in silicene (graphene). In other words, if we increase the nanotube diameter, we will see that the electrons start "losing" their masses, until they become Dirac massless fermions. Second, notice that for a given diameter, elec- trons in Si h-NT's are heavier, in magnitude, than electrons in CNT's. The top valence band and lowest conduction band are flatter than the corresponding ones in CNT's, which explains their mass difference.

We point out that the effective masses can be obtained from $\mathbf{k} \cdot \mathbf{p}$ theory as well. Ando ${ }^{24}$ derived the following dispersion relation for the nanotube band gap,

$$
E_{n \pm}(k)= \pm \gamma \sqrt{\kappa(n)^{2}+k^{\prime 2}},
$$

with

$$
\kappa=\frac{2 \pi}{L}\left(n-\frac{\nu}{3}\right), n=0, \pm 1, \pm 2, \ldots
$$

where $k^{\prime}$ is the wave vector measured from the $K$ point, $L$ is the magnitude of the chiral vector and $|\nu|=1$ for semiconducting nanotubes. Hence, the effective mass of the zig zag tubes is

$$
m_{ \pm}^{*}=\left.\frac{\hbar^{2}}{d^{2} E_{0 \pm} / d k^{\prime 2}}\right|_{k^{\prime}=0}= \pm \frac{4 \hbar^{2}}{3 \sqrt{3} \gamma_{0} a}\left(\frac{1}{d}\right) .
$$

This equation confirms what was stated above about the nanotube effective masses using TB theory, that is, $m^{*}$ decreases as the $d$ increases since it explicitly depends upon $d^{-1}$.

\section{Si g-NT's}

The second column of Fig. 3 shows the band structures of Si g-NT's with chiral vectors $(4,4),(6,6),(8,0)$ and $(12,0)$ obtained from the $s p^{3}$ model. Notice that for all graphs but the ones in the second column, the Fermi energy is at zero. From this figure, one notices that there is a proliferation of the number of bands in the Si g-NT's when compared to CNT's. In CNT's there is a total of $2 N$ bands. ${ }^{18}$ The factor of 2 comes from the $2 \times 2$ Hamiltonian, and $N$ bands come from the quantization of the wave vector $\mathbf{k}$ along the $\mathbf{K}_{1}$ direction. In Si g-NT's, there are $8 N$ bands; eight from the $8 \times 8$ Hamiltonian, and the other $N$ from the same quantization in $\mathbf{k}$. Hence, Si gNT's have four times the number of bands that CNT's have. Moreover, there are 4 electrons per Si atom and a total of $2 N$ atoms in the g-NT unit cell, hence, there are $4 \times 2 N=8 N$ electrons per unit cell. In particular, armchair and zig zag g-NT's, have $16 n$ bands and electrons per unit cell $(N=2 n)$. Table II summarizes some of the general characteristics of the h-NT and g-NT energy bands.

After wrapping the Si (111) sheet into a zig zag g$\mathrm{NT}$, we find that the $(8,0)$ tube is a semiconductor with a gap of $0.34 \mathrm{eV}$, and the $(12,0)$ is either a metal or a semiconductor of gap zero. The armchair $(4,4)$ and $(6,6)$ tubes could be metals or zero gap semiconductors, as well. In order to completely characterize the electronic behavior of the last three nanotubes, we need their density of states (DOS), which is not done here. However, due to the similarities with CNT's, we would expect them 
TABLE II. General characteristics of the h-NT and g-NT energy bands. Here, $\mathrm{e}^{-}$and u.c. refer to electrons and the nanotube unit cell, respectively.

\begin{tabular}{lcc}
\hline \hline & $\mathrm{h}-\mathrm{NT}\left(s p^{2}\right)$ & $\mathrm{g}-\mathrm{NT}\left(s p^{3}\right)$ \\
\hline $\mathrm{e}^{-}$per atom & 1 & 4 \\
atoms per u.c. & $2 N$ & $2 N$ \\
Number of bands & $2 N$ & $8 N$ \\
$\mathrm{e}^{-}$per u.c. & $2 N$ & $8 N$ \\
\hline \hline
\end{tabular}

to have a finite DOS at the Fermi level and thus, to be metals.

In general, Si g-NT's obey Hamada's rule like CNT's do. In order to understand this, we apply a similar reasoning used for CNT's. ${ }^{18}$ We first started with the Si (111) band structure (c.f. Fig. 2(e)), which has zero gap at the $K$ point of its Brillouin zone. Second, we sliced the $\mathrm{Si}$ (111) bands by quantizing the wavevector $\mathbf{k}$ along the $\mathbf{K}_{1}$ direction. Third, we used the zone-folding approximation to plot the Si g-NT bands. If any of the "slices" cuts the $K$ point then, the g-NT is a conductor, if it does not, then it is a semiconductor. In the language of group theory, the degeneracies caused by the vertical and the horizontal mirror plane symmetries in CNT's are not removed in the gear-like tube.

Next, let us compare our Si g-NT energy bands to the bands by Yang and Ni. ${ }^{7}$ From the previous comparisons of the Si sheets, we find better agreement between our calculations and the ab-initio ones by Yang and $\mathrm{Ni}^{7}$ when we use our $2 \mathrm{NN} s p^{3}$ TB model. Hence, we only compare our energy bands obtained from this theory to the $a b$ initio ones.

The Si g-NT energy bands by Yang and $\mathrm{Ni}^{7}$ are reproduced in the left-most column of the Fig 3. We find that all our band structures differ from their work. Whereas all our armchair tubes are metals or zero-gap semiconductors, their corresponding tubes are semiconductors. Our zig-zag tubes follow Hamada's rule, while theirs do not.

The authors argue that in zig zag Si g-NT's with small diameter like $(8,0)$, gaps do not occur because of the $\sigma^{*}-$ $\pi^{*}$ coupling. We think that this hypothesis is doubtful, since our theory does include this coupling and it does not close the gap of the small semiconducting tubes like $(8,0)$. Furthermore, band gaps tend to open and not to close when couplings are added. A possible explanation for the discrepancy is that the $a b$ initio calculation suffers from the density-functional-theory band-gap problem.

These significant differences between the TB and the $a b$ initio bands are quite surprising since both approaches coincide on the band structure of Si (111). We point out, though, that in their report, Yang and $\mathrm{Ni}^{7}$ do not explain the gap openings when the Si (111) sheet is rolled up to form Si g-NT's. ${ }^{7}$ One could think that curvature effects in Yang's results might open a gap in Si g-NT's, nonetheless, such gap should be, at most, of the order of meV. Consider a CNT and a Si g-NT with the same chirality, for instance, $\mathbf{C}_{h}=(12,0)$. It is known that the gap opening $\Delta E$ due to curvature in CNT's, is inversely proportional to it and it is close to $10 \mathrm{meV} \cdot{ }^{18,31}$ The transfer integral $|\gamma|$, can be used as an energy scale for the nanotubes, thus, $\Delta E \sim|\gamma| / d^{2}$. The ratio between the openings in CNT's and Si g-NT's is then given by

$$
\begin{aligned}
\frac{\Delta E_{\mathrm{Si}}}{\Delta E_{\mathrm{C}}} \sim \frac{\left|\gamma_{\mathrm{Si}}\right| / d_{\mathrm{Si}}^{2}}{\left|\gamma_{\mathrm{C}}\right| / d_{\mathrm{C}}^{2}}=\frac{\left|\gamma_{\mathrm{Si}}\right|}{\left|\gamma_{\mathrm{C}}\right|}\left(\frac{d_{\mathrm{C}}}{d_{\mathrm{Si}}}\right)^{2} \\
\Rightarrow \Delta E_{\mathrm{Si}} \sim \frac{\left|\gamma_{\mathrm{Si}}\right|}{\left|\gamma_{\mathrm{C}}\right|}\left(\frac{d_{\mathrm{C}}}{d_{\mathrm{Si}}}\right)^{2} \Delta E_{\mathrm{C}} .
\end{aligned}
$$

Consider a C- and a Si-NT with diameters $d_{\mathrm{C}}=$ $30 \AA$ and $d_{\mathrm{Si}}=46 \AA$, respectively. ${ }^{7,18}$ Substituting $\left|\gamma_{\mathrm{C}}\right|=$ $3.033 \mathrm{eV},\left|\gamma_{\mathrm{Si}}\right|=0.949 \mathrm{eV}$ and $\Delta E_{\mathrm{C}}=10 \mathrm{meV}^{7,18}$ in Eq. (13), we find that

$$
\Delta E_{\mathrm{Si}} \approx 1 \mathrm{meV}
$$

The band gap in the $(12,0) \mathrm{Si}$ g-NT of Yang and $\mathrm{Ni}^{7}$ (see band structure in the lower right corner of Fig. 3) is close to $200 \mathrm{meV}$ so, curvature cannot be responsible for the whole opening of the gap in Yang's nanotubes.

Our TB scheme, on the other hand, provides an explanation for the gap behavior in all g-NT's openings in terms of Hamada's rule. For example, our $(12,0) \mathrm{Si}$ gNT has, according to Hamada's rule, a zero gap since this corresponds to a zig zag tube with $n$ multiple of 3 .

\section{CONCLUSIONS}

The electronic properties of silicene, Si (111), Si hNT's and Si g-NT's were studied via a TB approach. We derived $s p^{3} s^{*}$ and $s p^{3}$ Hamiltonians up to $1 \mathrm{NN}$ and $2 \mathrm{NN}$, respectively.

We compared the band structures of Si (111) and of silicene to the one of graphene. Since all of these materials have in-plane symmetry, the $\pi$ bands are two-fold degenerate at $K$. We expect Si (111) and silicene to be either semiconductors of band gap zero or metals. Electrons in the neighborhood of the $K$ point should behave as Dirac massless fermions due to the presence of the Dirac cone in both structures. However, the Fermi velocities $v_{0}$ in Si $(111)\left(10^{4} \mathrm{~m} / \mathrm{s}\right)$ and in silicene $\left(10^{5} \mathrm{~m} / \mathrm{s}\right)$ are smaller than the one in graphene $\left(10^{6} \mathrm{~m} / \mathrm{s}\right)$. Electron tunneling between $\mathrm{Si}$ atoms is less favorable in $\mathrm{Si}$ (111) and silicene than in graphene because the $\pi$ interaction is weaker in the the first two cases.

Silicon h-NT's and Si g-NT's were compared to CNT's, as well. The band structure of Si h-NT's and of CNT's are similar two each other. Even though we performed calculations including $1 \mathrm{NN}$ and $2 \mathrm{NN}$, we found that the effect of the latter ones on the bands is negligible. This allowed us to make further approximations when calculating the band gap and effective masses of Si h-NT's. In the case of zig zag semiconductor Si h-NT's, the gap 
is inversely proportional to the tube diameter, as in zig zag CNT's, nonetheless, for a given diameter, Si h-NT's will have a smaller gap. The magnitude of the effective masses is also inversely proportional to their diameter, however, for a given diameter Si h-NT's have greater mass, which makes CNT's more suitable for transport properties. As far as Si g-NT's are concerned, we found that they follow Hamada's rule as CNT's do, even though they show different hybridizations.

Our calculations for all the Si-based materials considered here were compared to the $a b$ initio calculations performed by Yang and $\mathrm{Ni}^{7}$ When comparing silicene and Si (111), we found that the $2 \mathrm{NN} s p^{3}$ model is in better agreement with Yang's band structures than the 1NN $s p^{3} s^{*}$ model. For this reason, we chose the $s p^{3}$ model in order to reproduce the energy dispersions of Si nanotubes.

For Si h-NT's, our band structures agree with the ones obtained by Yang and $\mathrm{Ni}^{7}$ they all follow Hamada's rule. In contrast, they disagree for all Si g-NT's. Whereas our calculations show that these nanotubes also follow this rule, Yang's calculations do not. We emphasize that the $\sigma-\pi$ coupling does not close the gap for Si g-NT's with small diameters, contrary to the hypothesis of Yang and
Ni. ${ }^{7}$ Our calculations also show how critical it is to obtain accurate TB parameters for applications to Si sheets and nanotubes.

\section{ACKNOWLEDGMENTS}

We thank Yang and $\mathrm{Ni}$ for providing the data used in Figs. 2 and 3. The work was supported by an NSF CAREER award (NSF Grant No. 0454849), and by a Research Challenge grant from Wright State University and the Ohio Board of Regents.

\section{Appendix A: Tight-binding Hamiltonian}

In this appendix, we explicitly give the Hamiltonian of our model. The $s p^{3} s^{*}$ Hamiltonian has the form

$$
H(\mathbf{k})=\left[\begin{array}{ll}
h_{A A} & h_{A B} \\
h_{B A} & h_{B B}
\end{array}\right],
$$

where the $h_{i j}$ 's are $5 \times 5$ sub-matrices which are given as follows:

$$
\begin{aligned}
h_{A A}= & {\left[\begin{array}{ccccc}
(s / s)_{A A} & (s / x)_{A A} & (s / y)_{A A} & 0 & \left(s / s^{*}\right)_{A A} \\
& (x / x)_{A A} & (x / y)_{A A} & 0 & \left(x / s^{*}\right)_{A A} \\
& & (y / y)_{A A} & 0 & \left(y / s^{*}\right)_{A A} \\
& \dagger & & (z / z)_{A A} & 0 \\
& & & & \left(s^{*} / s^{*}\right)_{A A}
\end{array}\right], } \\
h_{A B}= & {\left[\begin{array}{lllll}
(s / s)_{A B} & (s / x)_{A B} & (s / y)_{A B} & (s / z)_{A B} & \left(s / s^{*}\right)_{A B} \\
(x / s)_{A B} & (x / x)_{A B} & (x / y)_{A B} & (x / z)_{A B} & \left(x / s^{*}\right)_{A B} \\
(y / s)_{A B} & (y / x)_{A B} & (y / y)_{A B} & (y / z)_{A B} & \left(y / s^{*}\right)_{A B} \\
(z / s)_{A B} & (z / x)_{A B} & (z / y)_{A B} & (z / z)_{A B} & \left(z / s^{*}\right)_{A B} \\
\left(s^{*} / s\right)_{A B} & \left(s^{*} / x\right)_{A B} & \left(s^{*} / y\right)_{A B} & \left(s^{*} / z\right)_{a c} & \left(s^{*} / s^{*}\right)_{A B}
\end{array}\right], } \\
h_{B B}= & {\left[\begin{array}{ccccc}
(s / s)_{A A}^{*} & -(s / x)_{A A}^{*} & -(s / y)_{A A}^{*} & 0 & \left(s / s^{*}\right)_{A A}^{*} \\
& (x / x)_{A A}^{*} & (x / y)_{A A}^{*} & 0 & \left(x / s^{*}\right)_{A A}^{*} \\
& & (y / y)_{A A}^{*} & 0 & \left(y / s^{*}\right)_{A A}^{*} \\
& \dagger & & (z / z)_{A A}^{*} & \left(z / s^{*}\right)_{A A}^{*} \\
& & & & \left(s^{*} / s^{*}\right)_{A A}^{*}
\end{array}\right], }
\end{aligned}
$$

and $h_{B A}=\left[h_{A B}\right]^{\dagger}$. In these equations, $h_{A A}$ and $h_{A B}$ correspond to the interaction between atoms at $A-A$ and $A-B$ lattice points, respectively. In the case of the $s p^{3}$ Hamiltonian, each sub-matrix is $4 \times 4$ instead. The matrix elements are given as follows: 


$$
\begin{aligned}
& (s / s)_{A A}=(s / s)_{B B}^{*}=E_{s}+(s s \sigma)_{2}^{A A} g_{13}(\mathbf{k}), \\
& (s / x)_{A A}=-(s / x)_{B B}^{*}=(s p \sigma)_{2}^{A A} g_{14}(\mathbf{k}), \\
& (s / y)_{A A}=-(s / y)_{B B}^{*}=(s p \sigma)_{2}^{A A} g_{15}(\mathbf{k}), \\
& (s / z)_{A A}=-(s / y)_{B B}^{*}=0, \\
& (x / x)_{A A}=(x / x)_{B B}^{*}=E_{p}+(p p \sigma)_{2}^{A A} g_{16}(\mathbf{k})+(p p \pi)_{2}^{A A} g_{17}(\mathbf{k}), \\
& (x / y)_{A A}=(x / y)_{B B}^{*}=\left[(p p \sigma)_{2}^{A A}-(p p \pi)_{2}^{A A}\right] g_{18}(\mathbf{k}), \\
& (x / z)_{A A}=(x / z)_{B B}^{*}=0 \text {, } \\
& (y / y)_{A A}=(y / y)_{B B}^{*}=E_{p}+(p p \sigma)_{2}^{A A} g_{19} \mathbf{k}+(p p \pi)_{2}^{A A} g_{20}(\mathbf{k}), \\
& (y / z)_{A A}=(y / z)_{B B}^{*}=0 \text {, } \\
& (z / z)_{A A}=(z / z)_{B B}^{*}=E_{p}+(p p \pi)_{2}^{A A} g_{25}(\mathbf{k}), \\
& (x / x)_{A B}=(p p \sigma)_{1}^{A B} g_{3}(\mathbf{k})+(p p \pi)_{1}^{A B} g_{4}(\mathbf{k}), \\
& (y / y)_{A B}=(p p \sigma)_{1}^{A B} g_{6}(\mathbf{k})+(p p \pi)_{1}^{A B} g_{4}(\mathbf{k}), \\
& (z / z)_{A B}=(p p \sigma)_{1}^{A B} g_{11}(\mathbf{k})+(p p \pi)_{1}^{A B} g_{12}(\mathbf{k}), \\
& (s / x)_{A B}=-(x / s)_{A B}=(s p \sigma)_{1}^{A B} g_{1}(\mathbf{k}), \\
& (s / y)_{A B}=-(y / s)_{A B}=(s p \sigma)_{1}^{A B} g_{2}(\mathbf{k}), \\
& (s / z)_{A B}=-(z / s)_{A B}=(s p \sigma)_{1}^{A B} g_{8}(\mathbf{k}), \\
& (x / y)_{A B}=(y / x)_{A B}=\left[(p p \sigma)_{1}^{A B}-(p p \pi)_{1}^{A B}\right] g_{5}(\mathbf{k}), \\
& (x / z)_{A B}=(z / x)_{A B}=\left[(p p \sigma)_{1}^{A B}-(p p \pi)_{1}^{A B}\right] g_{9}(\mathbf{k}), \\
& (y / z)_{A B}=(z / y)_{A B}=\left[(p p \sigma)_{1}^{A B}-(p p \pi)_{1}^{A B}\right] g_{10}(\mathbf{k}), \\
& \left(s^{*} / s^{*}\right)_{A A}=\left(s^{*} / s^{*}\right)_{B B}^{*}=E_{s^{*}}+\left(s^{*} s^{*} \sigma\right)_{2}^{A A} g_{13}(\mathbf{k}), \\
& \left(s^{*} / x\right)_{A A}=-\left(s^{*} / x\right)_{B B}^{*}=\left(s^{*} p \sigma\right)_{2}^{A A} g_{14}(\mathbf{k}), \\
& \left(s^{*} / y\right)_{A A}=-\left(s^{*} / y\right)_{B B}^{*}=\left(s^{*} p \sigma\right)_{2}^{A A} g_{15}(\mathbf{k}), \\
& \left(s^{*} / z\right)_{A A}=-\left(s^{*} / y\right)_{B B}^{*}=0 \text {, } \\
& \left(s / s^{*}\right)_{A A}=\left(s / s^{*}\right)_{B B}^{*}=\left(s s^{*} \sigma\right)_{2}^{A A} g_{13}(\mathbf{k}) \text {, } \\
& \left(s^{*} / x\right)_{A B}=-\left(x / s^{*}\right)_{A B}=\left(s^{*} p \sigma\right)_{1}^{A B} g_{1}(\mathbf{k}), \\
& \left(s^{*} / y\right)_{A B}=-\left(y / s^{*}\right)_{A B}=\left(s^{*} p \sigma\right)_{1}^{A B} g_{2}(\mathbf{k}), \\
& \left(s^{*} / z\right)_{A B}=-\left(z / s^{*}\right)_{A B}=\left(s^{*} p \sigma\right)_{1}^{A B} g_{8}(\mathbf{k}) \text {. }
\end{aligned}
$$




$$
\begin{aligned}
& g_{0}(\mathbf{k})=e^{i \mathbf{k} \cdot \boldsymbol{\delta}_{1}^{(1)}}+e^{i \mathbf{k} \cdot \boldsymbol{\delta}_{2}^{(1)}}+e^{i \mathbf{k} \cdot \boldsymbol{\delta}_{3}^{(1)}}, \\
& g_{1}(\mathbf{k})=-\frac{2 \sqrt{2}}{9}\left(e^{i \mathbf{k} \cdot \boldsymbol{\delta}_{1}^{(1)}}-\frac{1}{2} e^{i \mathbf{k} \cdot \boldsymbol{\delta}_{2}^{(1)}}-\frac{1}{2} e^{i \mathbf{k} \cdot \boldsymbol{\delta}_{3}^{(1)}}\right), \\
& g_{2}(\mathbf{k})=\sqrt{\frac{2}{3}}\left(e^{i \mathbf{k} \cdot \boldsymbol{\delta}_{2}^{(1)}}-e^{i \mathbf{k} \cdot \boldsymbol{\delta}_{3}^{(1)}}\right), \\
& g_{3}(\mathbf{k})=\frac{8}{9}\left(e^{i \mathbf{k} \cdot \boldsymbol{\delta}_{1}^{(1)}}+\frac{1}{4} e^{i \mathbf{k} \cdot \boldsymbol{\delta}_{2}^{(1)}} \frac{1}{4} e^{i \mathbf{k} \cdot \boldsymbol{\delta}_{3}^{(1)}}\right), \\
& g_{4}(\mathbf{k})=\frac{1}{9}\left(e^{i \mathbf{k} \cdot \boldsymbol{\delta}_{1}^{(1)}}+7 e^{i \mathbf{k} \cdot \boldsymbol{\delta}_{2}^{(1)}}+7 e^{i \mathbf{k} \cdot \boldsymbol{\delta}_{3}^{(1)}}\right), \\
& g_{5}(\mathbf{k})=-\frac{2}{3}\left(e^{i \mathbf{k} \cdot \boldsymbol{\delta}_{2}^{(1)}}+e^{i \mathbf{k} \cdot \boldsymbol{\delta}_{3}^{(1)}}\right), \\
& g_{6}(\mathbf{k})=\frac{2}{3}\left(e^{i \mathbf{k} \cdot \boldsymbol{\delta}_{2}^{(1)}}+e^{i \mathbf{k} \cdot \boldsymbol{\delta}_{3}^{(1)}}\right), \\
& g_{7}(\mathbf{k})=e^{i \mathbf{k} \cdot \boldsymbol{\delta}_{1}^{(1)}}+\frac{1}{3} e^{i \mathbf{k} \cdot \boldsymbol{\delta}_{2}^{(1)}}+\frac{1}{3} e^{i \mathbf{k} \cdot \boldsymbol{\delta}_{3}^{(1)}}, \\
& g_{8}(\mathbf{k})=-\frac{1}{3}\left(e^{i \mathbf{k} \cdot \boldsymbol{\delta}_{1}^{(1)}}+e^{i \mathbf{k} \cdot \boldsymbol{\delta}_{2}^{(1)}}+e^{i \mathbf{k} \cdot \boldsymbol{\delta}_{3}^{(1)}}\right), \\
& g_{9}(\mathbf{k})=-\frac{2 \sqrt{2}}{9}\left(e^{i \mathbf{k} \cdot \boldsymbol{\delta}_{1}^{(1)}}-\frac{1}{2} e^{i \mathbf{k} \cdot \boldsymbol{\delta}_{2}^{(1)}}-\frac{1}{2} e^{i \mathbf{k} \cdot \boldsymbol{\delta}_{3}^{(1)}}\right), \\
& g_{10}(\mathbf{k})=\frac{1}{3} \sqrt{\frac{2}{3}}\left(e^{i \mathbf{k} \cdot \boldsymbol{\delta}_{2}^{(1)}}-e^{i \mathbf{k} \cdot \boldsymbol{\delta}_{3}^{(1)}}\right) \\
& g_{11}(\mathbf{k})=\frac{1}{9}\left(e^{i \mathbf{k} \cdot \boldsymbol{\delta}_{1}^{(1)}}+e^{i \mathbf{k} \cdot \boldsymbol{\delta}_{2}^{(1)}}+e^{i \mathbf{k} \cdot \boldsymbol{\delta}_{3}^{(1)}}\right), \\
& g_{12}(\mathbf{k})=\frac{8}{9}\left(e^{i \mathbf{k} \cdot \boldsymbol{\delta}_{1}^{(1)}}+e^{i \mathbf{k} \cdot \boldsymbol{\delta}_{2}^{(1)}}+e^{i \mathbf{k} \cdot \boldsymbol{\delta}_{3}^{(1)}}\right)=\frac{8}{9}\left(e^{i \frac{k_{x} a}{\sqrt{3}}}+2 e^{-i \frac{k_{x} a}{2 \sqrt{3}}} \cos \frac{k_{y} a}{2}\right), \\
& g_{13}(\mathbf{k})=e^{i \mathbf{k} \cdot \boldsymbol{\delta}_{1}^{(2)}}+e^{i \mathbf{k} \cdot \boldsymbol{\delta}_{2}^{(2)}}+e^{i \mathbf{k} \cdot \boldsymbol{\delta}_{3}^{(2)}}+e^{i \mathbf{k} \cdot \boldsymbol{\delta}_{4}^{(2)}}+e^{i \mathbf{k} \cdot \boldsymbol{\delta}_{5}^{(2)}}+e^{i \mathbf{k} \cdot \boldsymbol{\delta}_{6}^{(2)}}, \\
& g_{14}(\mathbf{k})=\frac{\sqrt{3}}{2}\left(e^{i \mathbf{k} \cdot \boldsymbol{\delta}_{3}^{(2)}}-e^{i \mathbf{k} \cdot \boldsymbol{\delta}_{4}^{(2)}}+e^{i \mathbf{k} \cdot \boldsymbol{\delta}_{5}^{(2)}}-e^{i \mathbf{k} \cdot \boldsymbol{\delta}_{6}^{(2)}}\right) \\
& g_{15}(\mathbf{k})=e^{i \mathbf{k} \cdot \boldsymbol{\delta}_{1}^{(2)}}+e^{i \mathbf{k} \cdot \boldsymbol{\delta}_{2}^{(2)}}+\frac{1}{4} e^{i \mathbf{k} \cdot \boldsymbol{\delta}_{3}^{(2)}}+\frac{1}{4} e^{i \mathbf{k} \cdot \boldsymbol{\delta}_{4}^{(2)}}+\frac{1}{4} e^{i \mathbf{k} \cdot \boldsymbol{\delta}_{5}^{(2)}}-\frac{1}{4} e^{i \mathbf{k} \cdot \boldsymbol{\delta}_{6}^{(2)}}, \\
& g_{16}(\mathbf{k})=\frac{3}{4}\left(e^{i \mathbf{k} \cdot \boldsymbol{\delta}_{3}^{(2)}}+e^{i \mathbf{k} \cdot \boldsymbol{\delta}_{4}^{(2)}}+e^{i \mathbf{k} \cdot \boldsymbol{\delta}_{5}^{(2)}}+e^{i \mathbf{k} \cdot \boldsymbol{\delta}_{6}^{(2)}}\right), \\
& g_{17}(\mathbf{k})=e^{i \mathbf{k} \cdot \boldsymbol{\delta}_{1}^{(2)}}+e^{i \mathbf{k} \cdot \boldsymbol{\delta}_{2}^{(2)}}+\frac{3}{4} e^{i \mathbf{k} \cdot \boldsymbol{\delta}_{3}^{(2)}}+\frac{3}{4} e^{i \mathbf{k} \cdot \boldsymbol{\delta}_{4}^{(2)}}+\frac{3}{4} e^{i \mathbf{k} \cdot \boldsymbol{\delta}_{5}^{(2)}}+\frac{3}{4} e^{i \mathbf{k} \cdot \boldsymbol{\delta}_{6}^{(2)}}, \\
& g_{18}(\mathbf{k})=\frac{\sqrt{3}}{4}\left(-e^{i \mathbf{k} \cdot \boldsymbol{\delta}_{3}^{(2)}}-e^{i \mathbf{k} \cdot \boldsymbol{\delta}_{4}^{(2)}}+e^{i \mathbf{k} \cdot \boldsymbol{\delta}_{5}^{(2)}}+e^{i \mathbf{k} \cdot \boldsymbol{\delta}_{6}^{(2)}}\right) \\
& g_{19}(\mathbf{k})=e^{i \mathbf{k} \cdot \boldsymbol{\delta}_{1}^{(2)}}+e^{i \mathbf{k} \cdot \boldsymbol{\delta}_{2}^{(2)}}+\frac{1}{4} e^{i \mathbf{k} \cdot \boldsymbol{\delta}_{3}^{(2)}}+\frac{1}{4} e^{i \mathbf{k} \cdot \boldsymbol{\delta}_{4}^{(2)}}+\frac{1}{4} e^{i \mathbf{k} \cdot \boldsymbol{\delta}_{5}^{(2)}}+\frac{1}{4} e^{i \mathbf{k} \cdot \boldsymbol{\delta}_{6}^{(2)}}, \\
& g_{20}(\mathbf{k})=\frac{3}{4}\left(e^{i \mathbf{k} \cdot \boldsymbol{\delta}_{3}^{(2)}}+e^{i \mathbf{k} \cdot \boldsymbol{\delta}_{4}^{(2)}}+e^{i \mathbf{k} \cdot \boldsymbol{\delta}_{5}^{(2)}}+e^{i \mathbf{k} \cdot \boldsymbol{\delta}_{6}^{(2)}}\right), \\
& g_{21}(\mathbf{k})=g_{22}(\mathbf{k})=g_{23}(\mathbf{k})=0 \text {, } \\
& g_{24}(\mathbf{k})=e^{i \mathbf{k} \cdot \boldsymbol{\delta}_{1}^{(2)}}+e^{i \mathbf{k} \cdot \boldsymbol{\delta}_{2}^{(2)}}+e^{i \mathbf{k} \cdot \boldsymbol{\delta}_{3}^{(2)}}+e^{i \mathbf{k} \cdot \boldsymbol{\delta}_{4}^{(2)}}+e^{i \mathbf{k} \cdot \boldsymbol{\delta}_{5}^{(2)}}+e^{i \mathbf{k} \cdot \boldsymbol{\delta}_{6}^{(2)}},
\end{aligned}
$$

where the position vectors $\boldsymbol{\delta}_{j}^{(k)}$ are given in Eqs. (1) and $(2)$. 


$$
\begin{aligned}
& g_{0}(\mathbf{k})=e^{i \mathbf{k} \cdot \boldsymbol{\delta}_{1}^{(1)}}+e^{i \mathbf{k} \cdot \boldsymbol{\delta}_{2}^{(1)}}+e^{i \mathbf{k} \cdot \boldsymbol{\delta}_{3}^{(1)}}, \\
& g_{1}(\mathbf{k})=e^{i \mathbf{k} \cdot \boldsymbol{\delta}_{1}^{(1)}}-\frac{1}{2} e^{i \mathbf{k} \cdot \boldsymbol{\delta}_{2}^{(1)}}-\frac{1}{2} e^{i \mathbf{k} \cdot \boldsymbol{\delta}_{3}^{(1)}}, \\
& g_{2}(\mathbf{k})=\frac{\sqrt{3}}{2}\left(e^{i \mathbf{k} \cdot \boldsymbol{\delta}_{2}^{(1)}}-e^{i \mathbf{k} \cdot \boldsymbol{\delta}_{3}^{(1)}}\right), \\
& g_{3}(\mathbf{k})=e^{i \mathbf{k} \cdot \boldsymbol{\delta}_{1}^{(1)}}+\frac{1}{4} e^{i \mathbf{k} \cdot \boldsymbol{\delta}_{2}^{(1)}} \frac{1}{4} e^{i \mathbf{k} \cdot \boldsymbol{\delta}_{3}^{(1)}}, \\
& g_{4}(\mathbf{k})=\frac{3}{4}\left(e^{i \mathbf{k} \cdot \boldsymbol{\delta}_{2}^{(1)}}+e^{i \mathbf{k} \cdot \boldsymbol{\delta}_{3}^{(1)}}\right), \\
& g_{5}(\mathbf{k})=-\frac{\sqrt{3}}{4}\left(e^{i \mathbf{k} \cdot \boldsymbol{\delta}_{2}^{(1)}}-e^{i \mathbf{k} \cdot \boldsymbol{\delta}_{3}^{(1)}}\right), \\
& g_{6}(\mathbf{k})=\frac{3}{4}\left(e^{i \mathbf{k} \cdot \boldsymbol{\delta}_{2}^{(1)}}+e^{i \mathbf{k} \cdot \boldsymbol{\delta}_{3}^{(1)}}\right), \\
& g_{7}(\mathbf{k})=e^{i \mathbf{k} \cdot \boldsymbol{\delta}_{1}^{(1)}}+\frac{1}{4} e^{i \mathbf{k} \cdot \boldsymbol{\delta}_{2}^{(1)}}+\frac{1}{4} e^{i \mathbf{k} \cdot \boldsymbol{\delta}_{3}^{(1)}}, \\
& g_{8}(\mathbf{k})=g_{9}(\mathbf{k})=g_{10}(\mathbf{k})=g_{11}(\mathbf{k})=0, \\
& g_{12}(\mathbf{k})=e^{i \mathbf{k} \cdot \boldsymbol{\delta}_{1}^{(1)}}+e^{i \mathbf{k} \cdot \boldsymbol{\delta}_{2}^{(1)}}+e^{i \mathbf{k} \cdot \boldsymbol{\delta}_{3}^{(1)}}=e^{i \frac{k_{x} a}{\sqrt{3}}}+2 e^{-i \frac{k_{x} a}{2 \sqrt{3}}} \cos \frac{k_{y} a}{2}, \\
& g_{13}(\mathbf{k})=e^{i \mathbf{k} \cdot \boldsymbol{\delta}_{1}^{(2)}}+e^{i \mathbf{k} \cdot \boldsymbol{\delta}_{2}^{(2)}}+e^{i \mathbf{k} \cdot \boldsymbol{\delta}_{3}^{(2)}}+e^{i \mathbf{k} \cdot \boldsymbol{\delta}_{4}^{(2)}}+e^{i \mathbf{k} \cdot \boldsymbol{\delta}_{5}^{(2)}}+e^{i \mathbf{k} \cdot \boldsymbol{\delta}_{6}^{(2)}}, \\
& g_{14}(\mathbf{k})=\frac{\sqrt{3}}{2}\left(e^{i \mathbf{k} \cdot \boldsymbol{\delta}_{3}^{(2)}}-e^{i \mathbf{k} \cdot \boldsymbol{\delta}_{4}^{(2)}}+e^{i \mathbf{k} \cdot \boldsymbol{\delta}_{5}^{(2)}}-e^{i \mathbf{k} \cdot \boldsymbol{\delta}_{6}^{(2)}}\right), \\
& g_{15}(\mathbf{k})=e^{i \mathbf{k} \cdot \boldsymbol{\delta}_{1}^{(2)}}-e^{i \mathbf{k} \cdot \boldsymbol{\delta}_{2}^{(2)}}-\frac{1}{2} e^{i \mathbf{k} \cdot \boldsymbol{\delta}_{3}^{(2)}}+\frac{1}{2} e^{i \mathbf{k} \cdot \boldsymbol{\delta}_{4}^{(2)}}+\frac{1}{2} e^{i \mathbf{k} \cdot \boldsymbol{\delta}_{5}^{(2)}}-\frac{1}{2} e^{i \mathbf{k} \cdot \boldsymbol{\delta}_{6}^{(2)}}, \\
& g_{16}(\mathbf{k})=\frac{3}{4}\left(e^{i \mathbf{k} \cdot \boldsymbol{\delta}_{3}^{(2)}}+e^{i \mathbf{k} \cdot \boldsymbol{\delta}_{4}^{(2)}}+e^{i \mathbf{k} \cdot \boldsymbol{\delta}_{5}^{(2)}}+e^{i \mathbf{k} \cdot \boldsymbol{\delta}_{6}^{(2)}}\right), \\
& g_{17}(\mathbf{k})=e^{i \mathbf{k} \cdot \boldsymbol{\delta}_{1}^{(2)}}+e^{i \mathbf{k} \cdot \boldsymbol{\delta}_{2}^{(2)}}+\frac{1}{4} e^{i \mathbf{k} \cdot \boldsymbol{\delta}_{3}^{(2)}}+\frac{1}{4} e^{i \mathbf{k} \cdot \boldsymbol{\delta}_{4}^{(2)}}+\frac{1}{4} e^{i \mathbf{k} \cdot \boldsymbol{\delta}_{5}^{(2)}}+\frac{1}{4} e^{i \mathbf{k} \cdot \boldsymbol{\delta}_{6}^{(2)}}, \\
& g_{18}(\mathbf{k})=\frac{\sqrt{3}}{4}\left(-e^{i \mathbf{k} \cdot \boldsymbol{\delta}_{3}^{(2)}}-e^{i \mathbf{k} \cdot \boldsymbol{\delta}_{4}^{(2)}}+e^{i \mathbf{k} \cdot \boldsymbol{\delta}_{5}^{(2)}}+e^{i \mathbf{k} \cdot \boldsymbol{\delta}_{6}^{(2)}}\right), \\
& g_{19}(\mathbf{k})=e^{i \mathbf{k} \cdot \boldsymbol{\delta}_{1}^{(2)}}+e^{i \mathbf{k} \cdot \boldsymbol{\delta}_{2}^{(2)}}+\frac{1}{4} e^{i \mathbf{k} \cdot \boldsymbol{\delta}_{3}^{(2)}}+\frac{1}{4} e^{i \mathbf{k} \cdot \boldsymbol{\delta}_{4}^{(2)}}+\frac{1}{4} e^{i \mathbf{k} \cdot \boldsymbol{\delta}_{5}^{(2)}}+\frac{1}{4} e^{i \mathbf{k} \cdot \boldsymbol{\delta}_{6}^{(2)}}, \\
& g_{20}(\mathbf{k})=\frac{3}{4}\left(e^{i \mathbf{k} \cdot \boldsymbol{\delta}_{3}^{(2)}}+e^{i \mathbf{k} \cdot \boldsymbol{\delta}_{4}^{(2)}}+e^{i \mathbf{k} \cdot \boldsymbol{\delta}_{5}^{(2)}}+e^{i \mathbf{k} \cdot \boldsymbol{\delta}_{6}^{(2)}}\right), \\
& g_{21}(\mathbf{k})=g_{22}(\mathbf{k})=g_{23}(\mathbf{k})=g_{24}(\mathbf{k})=0 \text {, } \\
& g_{25}(\mathbf{k})=e^{i \mathbf{k} \cdot \boldsymbol{\delta}_{1}^{(2)}}+e^{i \mathbf{k} \cdot \boldsymbol{\delta}_{2}^{(2)}}+e^{i \mathbf{k} \cdot \boldsymbol{\delta}_{3}^{(2)}}+e^{i \mathbf{k} \cdot \boldsymbol{\delta}_{4}^{(2)}}+e^{i \mathbf{k} \cdot \boldsymbol{\delta}_{5}^{(2)}}+e^{i \mathbf{k} \cdot \boldsymbol{\delta}_{6}^{(2)}} .
\end{aligned}
$$

1 S. Iijima, Nature, 354, 56 (1991).

2 J. Goldberger, R. R. He, Y. F. Zhang, S. W. Lee, H. Q. Yan, H. J. Choi, and P. D. Yang, Nature 422, 599 (2003).

3 J. Zhang, C. J. Lu, and Z. Q. Li, Chin. J. Chem. Phys. 18, 113 (2005).

${ }^{4}$ G. C. Xi, Y. K. Liu, X. Y. Liu, X. Q. Wang, and Y. T. Qian, J. Phys. Chem. B 110, 14172 (2006).

5 Y. F. Zhukovskii, A. I. Popov, C. Balasubramanian, and S. Bellucci, J. Phys.: Condens. Matter 18, S2045 (2006).

6 S. Fagan, R. Baierle, R. Mota, A. J. da Silva, and A. Fazzio, Phys. Rev. B 61, 9994 (2000).

7 X. B. Yang and J. Ni, Phys. Rev. B 72, 195426 (2005).
${ }^{8}$ R. Q. Zhang, H. L. Lee, W. Li, and B. Teo, J. Phys. Chem. B 109, 8605 (2005).

${ }^{9}$ G. Seifert, T. Kohler, H. M. Urbassek, E. Hernandez, and T. Frauenheim, Phys. Rev. B 63, 193409 (2001).

10 J. Sha, J. J. Niu, X. Y. Ma, J. Xu, X. B. Zhang, Q. Yang, and D. Yang, Adv. Mater. 14, 1219 (2002).

11 S. Y. Jeong, J. Y. Kim, H. D. Yang, B. N. Yoon, S. H. Choi, H. K. Kang, C. W. Yang, and Y. H. Lee, Adv. Mater. 15, 1172 (2003).

${ }^{12}$ Y. W. Chen, Y. H. Tang, L. Z. Pei, and C. Guo, Adv. Mater. 17, 564 (2005).

13 Y. H. Tang, L. Z. Pei, Y. W. Chen, and C. Guo, Phys. Rev. Lett. 95, 116102 (2005). 
14 M. D. Crescenzi, P. Castrucci, M. Scarselli, M. Diociaiuti, P. S. Chaudhari, C. Balasubramanian, T. M. Bhave, and S. V. Bhoraskar, Appl. Phys. Lett. 86, 231901 (2005).

15 P. Castrucci, M. Scarselli, M. D. Crescenzi, M. Diociaiuti, P. S. Chaudhari, C. Balasubramanian, T. M. Bhave, and S. V. Bhoraskar, Thin Solid Films 508, 226 (2006).

16 B. Yan, G. Zhou, J. Wu, W. Duan, and B. L. Gu, Phys. Rev. B 73, 155432 (2006).

17 D. F. Perepichka and F. Rosei, Small 2, 22 (2006).

18 R. Saito, G. Dresselhaus, and M. S. Dresselhaus, Physical Properties of Carbon Nanotubes Imperial College Press, (Convent Garden, London, 1998).

19 P. Vogl, H. P. Hjalmarson, and J. D. Dow, J. Phys. Chem. Solids 44, 365 (1983).

${ }^{20}$ G. Grosso and C. Piermarocchi, Phys. Rev. B 51, 16772 (1995).

21 G. Klimeck, R. Bowen, T. Boykin, C. Salazar-Lazaro, T. Cwik, and A. Stoica, Superlattices Microstruct. 27, 77 (2000).

22 A. S. Martins, T. B. Boykin, G. Klimeck, and B. Koiller, Phys. Rev. B 72, 193204 (2005).
${ }^{23}$ K. S. Novoselov, A. K. Geim, S. V. Morozov, D. Jiang, M. I. Katsnelson, I. V. Grigorieva, S. V. Dubonos, and A. A. Firsov, Nature London 438, 197 (2005).

24 T. Ando, J. Phys. Soc. Jpn. 74, 777 (2005).

25 N. M. R. Peres, F. Guinea, and A. H. CastroNeto, Phys. Rev. B 73, 125411 (2006).

${ }^{26}$ C. Berger, Z. Song, X. Li, X. Wu, N. Brown, C. Naud, D. Mayou, T. Li, J. Hass, A. Marchenkov, E. Conrad, P. First and W. de Heer*, Science 312, 1191 (2006).

27 I. Milošević, B. Nikolić, M. Damnjanović, and M. Krčmar, J. Phys. A 31, 3625 (1998).

28 D. J. Chadi and M. L. Cohen, Phys. Status Solidi B 68, 405 (1975).

29 N. Hamada, S. I. Sawada, and A. Oshiyama, Phys. Rev. Lett. 68, 1579 (1992).

30 M. Dresselhaus, R. Jishi, G. Dresselhaus, D. Inomata, K. Nakao, and R. Saito, Mol. Mater. 4, 27 (1994).

31 S. Reich, C. Thomsen, and J. Maultzsch, Carbon Nanotubes: Basic Concepts and Physical Properties (WileyVCH, Germany, 2004). 